\title{
BMJ Open Programmes to support transitions in care for children and youth with complex care needs and their families: a scoping review protocol
}

\author{
Shelley Doucet (D) , ${ }^{1}$ Janet A Curran (D) , ${ }^{2}$ Sydney Breneol, ${ }^{2}$ Alison Luke (D) , \\ Emilie Dionne, ${ }^{3}$ Rima Azar, ${ }^{4}$ Amy E Reid (D) , ${ }^{1}$ Shelley McKibbon, ${ }^{5}$ \\ Amanda R Horsman (i) , ${ }^{6}$ Krystal Binns ${ }^{1}$
}

To cite: Doucet S, Curran JA, Breneol S, et al. Programmes to support transitions in care for children and youth with complex care needs and their families: a scoping review protocol. BMJ Open 2020;10:e033978. doi:10.1136/ bmjopen-2019-033978

- Prepublication history and additional material for this paper are available online. To view these files, please visit the journal online (http://dx.doi. org/10.1136/bmjopen-2019033978).

Received 30 August 2019 Revised 14 February 2020 Accepted 11 May 2020

Check for updates

(C) Author(s) (or their employer(s)) 2020. Re-use permitted under CC BY-NC. No commercial re-use. See rights and permissions. Published by BMJ.

For numbered affiliations see end of article.

Correspondence to

Dr Shelley Doucet;

sdoucet@unb.ca

\section{ABSTRACT}

Introduction Children and youth with complex care needs (CCNs) and their families experience many care transitions over their lifespan and are consequently vulnerable to the discontinuity or gaps in care that can occur during these transitions. Transitional care programmes, broadly defined as one or more intervention(s) or service(s) that aim to improve continuity of care, are increasingly being developed to address transitions in care for children and youth with CCNs. However, this literature has not yet been systematically examined at a comprehensive level. The purpose of this scoping review is to map the range of programmes that support transitions in care for children and youth with CCNs and their families during two phases of their lifespan: (1) up to the age of 19 years (not including their transition to adult healthcare) and (2) when transitioning from paediatric to adult healthcare.

Methods and analysis The Joanna Briggs Institute methodology for scoping reviews (ScR) will be used for the proposed scoping review. ScR are a type of knowledge synthesis that are useful for addressing exploratory research questions that aim to map key concepts and types of evidence on a topic and can be used to organise what is known about the phenomena. A preliminary search of PubMed was conducted in December 2018.

Ethics and dissemination Ethical approval is not required where this study is a review of the published and publicly reported literature. The research team's advisory council will develop a research dissemination strategy with goals, target audiences, expertise/leadership, resources and deadlines to maximise project outputs. The end-ofgrant activities will be used to raise awareness, promote action and inform future research, policy and practice on this topic.

\section{INTRODUCTION}

Children and youth with complex care needs (CCNs) and their families experience many care transitions over their lifespan and are consequently susceptible to lack of coordination between services or gaps in care that can occur during these transitions. Failure to successfully transition to a new care setting

\section{Strengths and limitations of this study}

- This paper describes a scoping review protocol that will map the range of programmes that support transitions in care for children and youth with complex care needs (CCNs) and their families during two phases of their lifespan: (1) up to the age of 19 years (not including their transition to adult healthcare) and (2) when transitioning from paediatric to adult healthcare.

- This scoping review will help us to better understand the programmes that have been described in the literature, and to explore patient and health system outcomes that have been measured and described.

- This review will result in the information that may be used to inform decision-making, practice and future research related to transitional care for children and youth with CCNs.

- Our team involves a range of stakeholders, including patients, families, researchers, clinicians, librarians and decision makers, who will provide input and contribute to decision-making during all phases of this review.

- The review includes only published literature, and studies will not be assessed for methodological quality.

has been shown to lead to higher utilisation of emergency departments, ${ }^{1}$ negative experiences of care for all those involved, ${ }^{2-5}$ poorer access to care, ${ }^{13-5}$ fragmentation of care ${ }^{16-8}$ deterioration of health due to lack of follow-up visits ${ }^{1}$ and highly demanding healthcare interventions. ${ }^{9-11}$ Furthermore, an unsuccessful transition from paediatric to adult services can negatively impact education and workplace achievement, which can further lead to risk-taking behaviours associated with morbidity and mortality beyond childhood and throughout the lifespan. ${ }^{12} 13$

Transitional care programmes, which include one or more intervention(s) or 
service(s) that target children, youth, or families with the goal of improving transitions in care for children or youth with CCNs, ${ }^{14}$ are continually being implemented to respond to transitional care needs for children and youth with CCNs. However, this literature has not been examined at a comprehensive level specific to the purpose of this scoping review, which is to map the range of programmes that support transitions in care for children and youth with CCNs and their families during two phases of their lifespan: (1) up to the age of 19 years (not including their transition to adult healthcare) and (2) when transitioning from paediatric to adult healthcare.

\section{BACKGROUND}

Approximately $19 \%$ of children have special healthcare needs that can result in limitations in their daily lives, ${ }^{15}$ with this number dramatically increasing over the past 40 years. ${ }^{16}$ Advances in healthcare and technology have increased the lifespan and quality of life of many children with CCNs, allowing more children to live at home, be a part of their community and live into adulthood. ${ }^{17} \mathrm{CCNs}$ refer to "multidimensional health and social care needs in the presence of a recognised medical condition or where there is no unifying diagnosis. They are individual and contextualised, they are continuing and dynamic and are present across a range of settings, impacted by healthcare structure' (Brenner, p1647). ${ }^{18}$ Although these children constitute a small percentage of the paediatric population, they represent a cohort using a high volume of health-related services (eg, tertiary healthcare, social and educational),${ }^{19}$ requiring a coordinated effort by their family and care team. ${ }^{20-22}$

Family members can play a large role throughout transitions in care because they remain a constant source of support, they are actively involved in caregiving, and can act as advocates where necessary. ${ }^{23}$ Caring for a child or youth with CCNs can require comprehensive home care, hospitalisations, unscheduled emergency room visits and countless appointments with specialists in and outside of primary care (eg, family physicians, physiotherapists and psychologists).${ }^{1724}$ In addition, these children, youth and their families experience many transitions in care (eg, between providers, between settings and between stages of illness) over their lifespan. With this, they are consequently susceptible to gaps in care that can occur during these transitions.

A preliminary search of PROSPERO, PubMed, the Cochrane Database of Systematic Reviews and the Joanna Briggs Institute (JBI) Database of Systematic Reviews and Implementation Reports was conducted and revealed that the topic of transitional care programmes (eg, from hospital to community, home care to respite care or from paediatric to adult healthcare) for children (0-19 years), youth (up to 25 years) and their families is a growing field. However, we found no evidence of systematic reviews or protocols that map the range of transitional care programmes for children/youth with CCNs and their families with the same objectives as this review. Recent reviews focus specifically on the barriers, needs, facilitators and/or outcomes of transitional care interventions. ${ }^{25-30}$ Furthermore, other reviews are tailored to specific illness presentations and needs (eg, spina bifida and mental health needs), ${ }^{41-38}$ specific transitions in care (eg, hospital to home) or solely on quantitative or empirical literature (eg, exclude qualitative studies). ${ }^{20} 2629$ For example, Watson and Warady targeted three specific health conditions (eg, cerebral palsy, autism spectrum disorders and diabetes) for their scoping review. ${ }^{39}$ Generalisable findings regarding transitional care programmes for children and youth with CCNs and their families have been difficult to extract due to their narrow focus on specific populations; heterogeneity of the interventions and outcome measures and limited descriptions of transitional care programmes. This field is quickly expanding with new evidence becoming available each year. A broad level scoping review is required to begin to make sense of this heterogeneous body of the literature. Mapping the current evidence regarding transitional care programmes for children and youth with CCNs and their families will assist with identifying gaps and create direction for future systematic reviews and research.

\section{REVIEW OBJECTIVES}

The objectives have been divided into two areas of transition programmes for children and youth with CCNs. The first objective is to map the range of programmes that have been reported in the literature to support children 19 years of age and under with CCNs and their families during transitions in care, not including their transition to adult healthcare. This would include, for example, transitions from hospital to home, home to school and home care to respite services. The second objective is to map the range of programmes that have been reported in the literature to support youth with CCNs and their families specific to their transition from paediatric to adult healthcare.

\section{REVIEW QUESTIONS}

1. What programmes have been reported in the literature to support children 19 years of age and under with CCNs and their families during transitions in care, not including their transition to adult healthcare?

2. What programmes have been reported in the literature to support youth with CCNs and their families as they transition from paediatric to adult healthcare?

\section{METHODS}

The proposed scoping review will be conducted in accordance with the JBI methodology for scoping reviews $(\mathrm{ScR}) .{ }^{40}{ }^{41} \mathrm{ScR}$ are a type of knowledge synthesis that are useful for addressing exploratory research questions that aim to map key concepts and types of evidence on a 
topic and can be used to organise what is known about the phenomena. ${ }^{40-42}$ Given the current state of the literature in this area, a scoping review is needed to better understand the range of transitional care programmes that have been described in the literature, and to explore patient and health system outcomes that have been measured and described. This would result in an organising framework that could help summarise the range of research activities in the field and identify gaps and future research questions.

Briefly, the steps within the JBI scoping review framework are: (1) defining and aligning the objective/s and question/s; (2) developing and aligning the inclusion criteria with the objective/s and question/s; (3) describing the planned approach to evidence searching, selection, extraction and charting; (4) searching for the evidence; (5) selecting the evidence; (6) extracting the evidence; (7) charting the evidence; (8) summarising the evidence in relation to the objective/s and question/s and (9) consultation of information scientists, librarians and/ or experts throughout. ${ }^{41}$ Peters $e t$ al state that ScR begin by developing an a priori protocol, and have a broad scope related to their inclusion criteria which correspond with the target population, concept and context. ${ }^{41}$ This information is presented below.

Prior to commencing the review, we will establish an Advisory Council comprised of key stakeholders, such as researchers, librarians and patients (at least one from each participating province), to oversee project milestones. Our team has adopted the term patient to refer to individuals with personal experience of a health issue and informal caregivers. ${ }^{43}$ Given our focus on children and youth and their families, we have engaged parents who have a child with CCNs as members of our research team, hereafter referred to as patients for clarity.

\section{Search strategy}

The search strategy will aim to locate published articles. An initial limited search of PubMed and CINAHL was undertaken in December 2018 to identify articles on the topic of interest. The concepts identified in titles and abstracts of relevant articles, and the index terms used to describe the articles were leveraged to develop a full search strategy in each of the included databases (see online supplementary appendix I). To ensure that all relevant literature is identified, the search strategy will undergo the Peer Review of Electronic Search Strategies Guideline Statement. ${ }^{44}$ Two library scientists will complete this process, with ARH developing the initial search strategy and SM completing the peer-review process. The search strategy, including all identified keywords and index terms, will be adapted for each identified database. The reference list of all articles selected for inclusion in the review will be screened manually for additional articles of interest.

\section{Information sources}

The databases to be searched include PubMed, CINAHL, ERIC, PsycINFO and Social Work Abstracts. Given the broad and international scope of this review, we will not include a search of the grey literature. This will be the focus of a future study.

\section{Inclusion criteria}

Participants: This review will consider all the literature on children and youth with CCNs (0-19 years) who have experienced transitions in care prior to transitioning from paediatric to adult healthcare, as well as youth who are engaged in transition programmes to support their transition from paediatric to adult healthcare. Families of children or youth with CCNs will also be included (eg, parents, guardians or other members caring for a child or youth). CCNs refer to 'multidimensional health and social care needs in the presence of a recognised medical condition or where there is no unifying diagnosis' (Brenner, p1647). ${ }^{18}$ Children and youth with specific health conditions as well as a broader paediatric population with CCNs will be included. Articles will also be included if the target population is being cared for under paediatric care services. Programmes or services that are designed to support children and youth without CCNs and individuals residing in long-term care facilities will not be included.

Concept: The concept of interest for this review is transitional care programmes. For the purpose of this review, transitional care will involve any movement of the child/youth and family between care settings (home to school/childcare and vice versa), care providers (primary care to specialised clinic) or services (home care to respite care; paediatric to adult services). Articles that do not explicitly state their intent to support transitions in care will be excluded. A programme will be defined as one or more interventions or services that target children, youth or their families with the goal of improving transitions in care for children or youth with CCNs. Programmes may be delivered in person or by distance by either a trained or an untrained provider (eg, lay persons).

Context: This review will consider articles where transitional care programmes are delivered anywhere in the child/youth's home community (eg, child/youth's home, school/childcare), neighbouring communities (eg, for children/youth who live in remote and rural areas) or primary hospital/institution (eg, primary care provider's office and tertiary care facility). Articles describing transitional care programmes that are delivered by a range of different modalities (eg, e-health and clinic based) will be considered for inclusion. However, literature sources describing programmes that are delivered exclusively within a hospital setting (such as intrafacility handover) with no community component will not be included. There will be no geographic or temporal limitations placed on this review to allow for the examination of any potential trends in transitional care programmes across time. 


\section{Types of sources}

This scoping review will consider all types of published literature sources, including experimental and quasiexperimental study designs, such as randomised controlled trials, non-randomised controlled trials, before and after studies and interrupted time-series studies. In addition, analytical observational studies including prospective and retrospective cohort studies, case-control studies and analytical cross-sectional studies will be considered for inclusion. This review will also consider descriptive observational study designs including case series, individual case reports and descriptive cross-sectional studies. Articles using qualitative research designs will also be considered, including but not limited to, designs such as phenomenology, grounded theory, ethnography, qualitative description, action research and feminist research. Text and descriptive papers will also be considered for inclusion in this scoping review if sufficient information is provided to discern programme characteristics. Systematic, scoping and literature reviews will not be considered for inclusion in this review; however, the reference lists of relevant reviews will be hand searched for additional articles. Articles published in English and French will be included.

\section{Study selection}

Screening for study selection will occur in two stages: title/abstract and then full text. Prior to the screening, all identified citations will be collated in Mendeley to correct citation errors and remove duplicates. All citations will be uploaded into Covidence Systematic Review Software and any undetected duplicates will be removed. ${ }^{45} 46$

To ensure our inclusion and exclusion criteria are uniformly applied, the initial screening will be piloted by two independent reviewers using 5-10 references from our initial search followed by a consensus meeting. On final adjustments, the official title and abstract screening will begin. Titles and abstracts will be screened by two independent reviewers for assessment against the inclusion criteria for the review. For the second stage of screening, all potentially relevant articles identified during the title and abstract screening will be retrieved in full text to be imported into the Covidence Software. ${ }^{45}$ The full text of selected citations will be assessed in detail against the inclusion criteria by two independent reviewers. The reasons for exclusion of full-text articles will be recorded and reported. Any disagreements that arise between the reviewers at each stage of the study selection process will be resolved through discussion. If consensus cannot be achieved, a third reviewer will be consulted. The results of the search will be reported in full, following the Preferred Reporting Items for Systematic Reviews and Meta-Analyses (PRISMA) extension for ScR and presented in a PRISMA flow diagram. ${ }^{42} 47$

\section{Data extraction}

Data will be extracted from the articles included in this scoping review by two independent reviewers using a data extraction tool developed by the research team. Extracted data will include specific details about the population, concept, context, study methods and key findings relevant to each of the review objectives. A draft extraction table is provided (see online supplementary appendix II). Articles will be sorted and organised under the two transitions of interest as outlined in our research questions: (1) transitions in care up to the age of 19 years and not including transitioning to adult healthcare and (2) transitions in care from paediatric to adult healthcare services. The data extraction table will be tested by two reviewers with three articles to ensure all relevant information is being captured, followed by a meeting to discuss any modification and revision deemed necessary. Other information that may be deemed relevant by the research team will also be extracted. Modifications will be detailed in the full scoping review report. Any disagreements that arise between the reviewers will be resolved through discussion to achieve consensus. If consensus cannot be achieved, a third reviewer will be consulted to make the final decision. Authors of included articles will be contacted to request missing or additional data, where required.

\section{Data presentation}

The results of this review will be presented separately by review objective. For each objective, a narrative summary will accompany the tabulated results and will describe how the data relate to the review's overall objectives and questions. This will include the following categories: article identification (eg, author/year); article characteristics (eg, objective and population); programme design (eg, outcomes and focus of transition) and the results (eg, barriers and enablers). We will use the Theory, Model and Framework Comparison and Selection Tool to identify the appropriate framework(s) to organise and categorise extracted intervention and programme descriptions. ${ }^{48}$ The data that are presented in the final report will be reflective of the information collected using the data extraction tool (online supplementary appendix II). Further, all information for this scoping review will be presented following the PRISMA-ScR Guidelines. ${ }^{42} \mathrm{We}$ also used the PRISMA-P reporting guidelines to promote transparency and to ensure that we addressed all the components that are applicable for a scoping review. ${ }^{47}$

\section{Patient and public involvement}

Our research team includes a range of stakeholders who will contribute to decision-making during all phases of this review. We will engage a range of stakeholders (beyond our Advisory Council) to provide input on our preliminary findings. This process will begin by identifying key authors from included articles and inviting them to comment on our preliminary findings, either during a telephone interview or by email. Next, a consensus meeting will be held with important knowledge users (eg, patients, clinicians and government) to solicit initial feedback on our findings to provide greater context and understanding of the data. Feedback from our research 
experts and knowledge users will be incorporated into our final report.

\section{ETHICS AND DISSEMINATION}

Ethical approval is not required where this study is a review of the published and publicly reported literature. In terms of dissemination, the research team's advisory council will develop a research dissemination strategy with goals, target audiences, expertise/leadership, resources and deadlines to maximise project outputs. The end-ofgrant activities will be used to raise awareness, promote action and inform future research, policy and practice on this topic. Examples of activities include presenting two conference presentations per objective (eg, at the Canadian Association for Health Services and Policy Research conference and the North American Primary Care Research Group conference); presenting to the Canadian Pediatric Society; sharing findings on the JBI database of systematic reviews and implementation reports, publishing one open access publication per objective (eg, BMC Public Health journal); participate in a consensus meeting, initiated through the Advisory Council, share findings through our websites and social media channels and policy briefs.

\section{Author affiliations}

${ }^{1}$ Department of Nursing and Health Sciences, University of New Brunswick, Saint John, New Brunswick, Canada

${ }^{2}$ School of Nursing, Faculty of Health, Dalhousie University, Halifax, Nova Scotia, Canada

${ }^{3}$ St. Mary's Research Centre \& Family Medicine, McGill University, Montreal, Quebec, Canada

${ }^{4}$ Department of Psychology, Mount Allison University, Sackville, New Brunswick, Canada

${ }^{5}$ W.K. Kellogg Health Sciences Library, Dalhousie University, Halifax, Nova Scotia, Canada

${ }^{6}$ Interdisciplinary Studies, University of New Brunswick, Saint John, New Brunswick, Canada

Acknowledgements We would like to thank all the members of our research team for their contribution to the development of this protocol: Roger Chafe, Kevin Chan, John Gordon, Jennifer Splane, Jocelyn Vine, Fredrick Burge, Stacy Burgess, Patricia Charlton, Jan Willem Gorter, Lisa Keeping-Burke, Andrew Lynk, Ruth MartinMisener, Jessie-Lee Maclsaac, Dan Nagel, Angela Riveroll, Anja Salijevic, Katherine Kelly, Julie Clegg, Genevieve Loughlin, Lisa Morissette, Cathie Scott, Judy Roy, Lisa Stromquist, Jamie Clark, Amy Grant, Sarah MacDougall and Jacklynn Pidduck.

Contributors All authors (SD, JAC, SB, AL, ED, RA, AER, SM, ARH and KB) contributed to the project including the design, preparation and editing of the scoping review protocol. SD, SB and AER developed the initial draft of the protocol and, after several iterations with significant input from authors JAC, AL, ED, RA, ARH, SM and KB, all team members approved the final manuscript that was submitted for publishing. In consultation with the research team, an experienced librarian (ARH) developed the search strategy for the scoping review and a second librarian (SM) conducted a peer review of the search strategy. All authors will contribute to the data synthesis and writing of the final scoping review.

Funding This work is funded from an operating grant from the Canadian Institutes of Health Research (CIHR) Pan-Canadian Strategy for Patient-Oriented Research (SPOR) Network in Primary and Integrated Health Care Innovations (PIHCI) Knowledge Synthesis Grant. Matching partners that contributed either cash or in-kind support for this grant include: New Brunswick Health Research Foundation, Eastern Health/Janeway Children's Hospital, Maritime SPOR SUPPORT Unit, Dalhousie University, IWK Health Centre, and Prince Edward Island PIHCI Network.

Competing interests None declared.
Patient and public involvement Patients and/or the public were involved in the design, or conduct, or reporting, or dissemination plans of this research. Refer to the Methods section for further details.

Patient consent for publication Not required.

Provenance and peer review Not commissioned; externally peer reviewed.

Open access This is an open access article distributed in accordance with the Creative Commons Attribution Non Commercial (CC BY-NC 4.0) license, which permits others to distribute, remix, adapt, build upon this work non-commercially, and license their derivative works on different terms, provided the original work is properly cited, appropriate credit is given, any changes made indicated, and the use is non-commercial. See: http://creativecommons.org/licenses/by-nc/4.0/.

ORCID iDs

Shelley Doucet http://orcid.org/0000-0003-4420-8199

Janet A Curran http://orcid.org/0000-0001-9977-0467

Alison Luke http://orcid.org/0000-0002-2800-579X

Amy E Reid http://orcid.org/0000-0002-7808-8247

Amanda R Horsman http://orcid.org/0000-0002-8281-2752

\section{REFERENCES}

1 Committee on Improving the Health, Safety, and Well-Being of Young Adults, Board on Children, Youth, and Families, Institute of Medicine, National Research CouncilBonnie RJ, Stroud C, Breiner H, eds. Investing in the health and well-being of young adults. Washington (DC): National Academies Press (US), 2015. https://www.ncbi.nlm. nih.gov/books/NBK284794/

2 Heath G, Farre A, Shaw K. Parenting a child with chronic illness as they transition into adulthood: a systematic review and thematic synthesis of parents' experiences. Patient Educ Couns 2017;100:76-92.

3 McManus F, Sacadura C, Clark DM. Why social anxiety persists: an experimental investigation of the role of safety behaviours as a maintaining factor. J Behav Ther Exp Psychiatry 2008;39:147-61 .

4 Rutishauser C, Sawyer SM, Ambresin A-E. Transition of young people with chronic conditions: a cross-sectional study of patient perceptions before and after transfer from pediatric to adult health care. Eur J Pediatr 2014;173:1067-74.

5 van Staa AL, Jedeloo S, van Meeteren J, et al. Crossing the transition chasm: experiences and recommendations for improving transitional care of young adults, parents and providers. Child Care Health Dev 2011;37:821-32.

6 Guavin F-P, Wilson M, Lavis J, et al. Improving care and support for people with multiple chronic health conditions in Ontario. Available: https://www.mcmasterforum.org/docs/default-source/productdocuments/citizen-briefs/care-for-people-with-multiple-chronichealth-conditions-cb.pdf?sfvrsn $=0$

7 Rich EC, Lipson D, Libersky J, et al. Organizing care for complex patients in the patient-centered medical home. Ann Fam Med 2012;10:60-2.

8 Kodner DL. All together now: a conceptual exploration of integrated care. Healthc Q 2009;13 Spec No:6-15.

9 Paré G, Raymond L, de Guinea AO, et al. Barriers to organizational adoption of EMR systems in family physician practices: a mixedmethods study in Canada. Int J Med Inform 2014;83:548-58.

10 Hwang W, Chang J, Laclair M, et al. Effects of integrated delivery system on cost and quality. Am J Manag Care 2013;19:175-84.

11 Nolte $E$, Pitchforth $E$. What is the evidence on the economic impacts of integrated care? Available: http://www.euro.who.int/_data/assets/ pdf_file/0019/251434/What-is-the-evidence-on-the-economicimpacts-of-integrated-care.pdf

12 Park MJ, Paul Mulye T, Adams SH, et al. The health status of young adults in the United States. J Adolesc Health 2006;39:305-17.

13 Park MJ, Scott JT, Adams SH, et al. Adolescent and young adult health in the United States in the past decade: little improvement and young adults remain worse off than adolescents. $J$ Adolesc Health 2014;55:3-16.

14 Rosen DS, Blum RW, Britto M, et al. Transition to adult health care for adolescents and young adults with chronic conditions: position paper of the Society for adolescent medicine. J Adolesc Health 2003;33:309-11.

15 United States Census Bureau. 2018 National Survey of Children's Health Topical Data and Input Files. United States Census Bureau, Washington, DC, 2018. https://www2.census.gov/programs-surveys/ nsch/datasets/2018/nsch_2018_topical_Stata.zip 
16 Perrin JM, Anderson LE, Van Cleave J. The rise in chronic conditions among infants, children, and youth can be Met with continued health system innovations. Health Aff 2014;33:2099-105.

17 Kuo DZ, Cohen E, Agrawal R, et al. A national profile of caregiver challenges among more medically complex children with special health care needs. Arch Pediatr Adolesc Med 2011;165:1020-6.

18 Brenner M, Kidston C, Hilliard C, et al. Children's complex care needs: a systematic concept analysis of multidisciplinary language. Eur J Pediatr 2018;177:1641-52.

19 Cohen E, Berry JG, Camacho X, et al. Patterns and costs of health care use of children with medical complexity. Pediatrics 2012;130:e1463-70.

20 Breneol S, Belliveau J, Cassidy C, et al. Strategies to support transitions from hospital to home for children with medical complexity: a scoping review. Int J Nurs Stud 2017;72:91-104.

21 Berry JG, Agrawal R, Kuo DZ, et al. Characteristics of hospitalizations for patients who use a structured clinical care program for children with medical complexity. J Pediatr 2011;159:284-90.

22 Cohen E, Lacombe-Duncan A, Spalding K, et al. Integrated complex care coordination for children with medical complexity: a mixedmethods evaluation of tertiary care-community collaboration. BMC Health Serv Res 2012;12:366.

23 Jeffs L, Saragosa M, Law MP, et al. The role of caregivers in interfacility care transitions: a qualitative study. Patient Prefer Adherence 2017;11:1443-50.

24 Cohen E, Berry JG, Sanders L, et al. Status Complexicus? the emergence of pediatric complex care. Pediatrics 2018;141:S202-11. 25.

25 Bhawra J, Toulany A, Cohen E, et al. Primary care interventions to improve transition of youth with chronic health conditions from paediatric to adult healthcare: a systematic review. BMJ Open 2016;6:e011871.

26 Campbell F, Biggs K, Aldiss SK, et al. Transition of care for adolescents from paediatric services to adult health services. Cochrane Database Syst Rev 2016;4:CD009794.

27 Coyne B, Hallowell SC, Thompson M. Measurable outcomes after transfer from pediatric to adult providers in youth with chronic illness. J Adolesc Health 2017;60:3-16.

28 Gabriel P, McManus M, Rogers K, et al. Outcome evidence for structured pediatric to adult health care transition interventions: a systematic review. J Pediatr 2017;188:e15:263-9.

29 Hart LC, Patel-Nguyen SV, Merkley MG, et al. An evidence map for interventions addressing transition from pediatric to adult care: a systematic review of systematic reviews. J Pediatr Nurs 2019;48:18-34

30 Acuña Mora M, Moons P, Sparud-Lundin C, et al. Assessing the level of evidence on transfer and transition in young people with chronic conditions: protocol of a scoping review. Syst Rev 2016;5:166.

31 Binks JA, Barden WS, Burke TA, et al. What do we really know about the transition to adult-centered health care? A focus on cerebral palsy and spina bifida. Arch Phys Med Rehabil 2007;88:1064-73.
32 While A, Forbes A, Ullman R, et al. Good practices that address continuity during transition from child to adult care: synthesis of the evidence. Child Care Health Dev 2004;30:439-52.

33 Rapley P, Davidson PM. Enough of the problem: a review of time for health care transition solutions for young adults with a chronic illness. J Clin Nurs 2010;19:313-23.

34 Crawford MJ, de Jonge E, Freeman GK, et al. Providing continuity of care for people with severe mental illness- a narrative review. Soc Psychiatry Psychiatr Epidemiol 2004;39:265-72.

35 Di Rezze B, Nguyen T, Mulvale G, et al. A scoping review of evaluated interventions addressing developmental transitions for youth with mental health disorders. Child Care Health Dev 2016;42:176-87.

36 Embrett MG, Randall GE, Longo CJ, et al. Effectiveness of health system services and programs for youth to adult transitions in mental health care: a systematic review of academic literature. Adm Policy Ment Health 2016;43:259-69.

37 Mulvale GM, Nguyen TD, Miatello AM, et al. Lost in transition or translation? care philosophies and transitions between child and youth and adult mental health services: a systematic review. J Ment Health 2016;28:1-10.

38 Paul M, Street C, Wheeler N, et al. Transition to adult services for young people with mental health needs: a systematic review. Clin Child Psychol Psychiatry 2015;20:436-57.

39 Watson AR, Warady BA. Transition from pediatric to adult-centered care. Dial Transplant 2011;40:156-8.

40 Joanna Briggs Institute. Joanna Briggs Institute Reviewer's Manual: Methodology for JBI Scoping Reviews, 2015. Available: http://joannabriggs.org/assets/docs/sumari/Reviewers-Manual Methodology-for-JBI-Scoping-Reviews_2015_v2.pdf

41 Peters MDJ, Godfrey CM, Khalil H, et al. Guidance for conducting systematic scoping reviews. Int J Evid Based Healthc 2015;13:141-6.

42 Tricco AC, Lillie E, Zarin W, et al. PRISMA extension for scoping reviews (PRISMA-ScR): checklist and explanation, 2018. Available: http://annals.org/article.aspx?doi=10.7326/M18-0850

43 Canadian Institute of Health Research. Strategy for patient-oriented research patient engagement framework, 2014. Available: http:// www.cihr-irsc.gc.ca/e/documents/spor_framework-en.pdf

44 McGowan J, Sampson M, Salzwedel DM, et al. PRESS Peer Review of Electronic Search Strategies: 2015 Guideline Statement. J Clin Epidemiol 2016;75:40-6.

45 Covidence Systematic Review Software. Covidence systematic review software, 2018. Available: https://www.covidence.org/

46 Tricco AC, Lillie E, Zarin W, et al. A scoping review on the conduct and reporting of scoping reviews. BMC Med Res Methodol 2016;16:15

47 Moher D, Shamseer L, Clarke M, et al. Preferred reporting items for systematic review and meta-analysis protocols (PRISMA-P) 2015 statement. Syst Rev 2015;4:1.

48 Birken SA, Rohweder CL, Powell BJ, et al. T-CaST: an implementation theory comparison and selection tool. Implement Sci 2018;13:143 